\section{TOMMorrow's AD marker}

\author{
By Lev Osherovich, Senior Writer
}

Since its discovery as an Alzheimer's disease risk factor in the early 1990s, mutations in APOE have been implicated in up to 50\% of AD cases. Now, the researcher who first detected APOE's role in AD is touting a new theory: he points to APOE's immediate chromosomal neighbor, the gene for a mitochondrial protein called TOMM40, as a key contributor to the neurodegenerative disease.

Allen Roses, director of the Deane Drug Discovery Institute at Duke University, presented his TOMM40 (translocase of outer mitochondrial membrane 40 homolog) findings at the International Conference on Alzheimer's Disease (ICAD) in Vienna in July. Although the work has not yet been published, Roses is already heading a company, Zinfandel Pharmaceuticals Inc., which plans to commercialize the discovery.

Roses formerly was SVP of genetics research and pharmacogenomics at GlaxoSmithKline plc. During an earlier stint at Duke, he led a team that identified the E4 variant of APOE as the most powerful risk factor for the most common, sporadic form of AD. Apolipoprotein E (APOE) is a cholesterol-ferrying protein involved in lipid metabolism in the cardiovascular system and the brain. Several genetic variants of APOE occur in humans, each with different biochemical properties and effects on AD.

$\mathrm{E} 4$ carriers have an earlier age of onset for $\mathrm{AD}$ and a more aggressive course of disease compared with carriers of other APOE alleles. $\mathrm{E} 4$ carriers also have proven more resistant to several investigational therapies than noncarriers, leading companies to stratify such patients into separate arms in ongoing trials. ${ }^{1}$

Roses' earlier discovery of E4's link to AD "is one of the most important and reproducible genetic findings for any complex genetic disorder," said Steven Paul, president of Lilly Research Laboratories, the research arm of Eli Lilly and Co., and SVP of science and technology at Eli Lilly.

However, some patients without the E4 allele develop disease earlier and respond more poorly to therapy than others. Geneticists turned to complex methods of genomic analysis to identify other $\mathrm{AD}$ susceptibility loci but thus far have not found others with effects as powerful as APOE.

Roses now thinks he has found one and says his data suggest that
TOMM40 is a major susceptibility gene that accelerates disease independently of E4.

Indeed, the findings may help explain why some carriers of a common, benign form of APOE called E3 develop AD earlier than others.

Because the primary causes of $\mathrm{AD}$ and the mechanism of APOE's role in the disease are hotly debated, academic and industry researchers have met Roses' claims about TOMM40 with caution. The real test of whether TOMM40 status can predict rapid disease progression will be a prophylactic $\mathrm{AD}$ trial using TOMM40 as a diagnostic marker-a trial for which Zinfandel is already recruiting patients.

\section{Genetic detectives}

Roses told SciBX that studies of TOMM40 began during his tenure at GSK, where he was in charge of hunting down AD risk genes. Genomewide association studies by GSK and others hinted that the region around $A P O E$ contributed more to $\mathrm{AD}$ risk than could be accounted for by $\mathrm{E} 4$ alone, leading him to suspect that nearby genes were hidden players in pathogenesis.

In 2005, Roses' team at GSK sequenced the chromosomal region encompassing TOMM40 and $A P O E$ in patients and controls. They used phylogenetic analysis to arrange individual sequences into related groups that reflected the evolutionary history of the chromosomal region.

"We came up with a very interesting region of about 10,000 base pairs" with two distinct types of sequence, said Roses. All E4 carriers, as well as a fraction of $\mathrm{E} 3$ carriers, had one variant of the region, whereas most E3 carriers had the other variant.

However, it was not clear which specific mutations within the variable region were most closely associated with AD, so GSK dropped the project. Shortly thereafter, Roses left the company to head drug discovery at Duke. Between jobs, he personally financed the sequencing of additional $\mathrm{AD}$ cases and controls and narrowed down the most statistically powerful effect on $\mathrm{AD}$ risk to a region within TOMM40.

Roses' team at Duke then discovered a sequence length variant in a noncoding region of TOMM40 that strongly correlated with disease. The team found that $\mathrm{E} 4$ carriers always had the longer, high-risk form of TOMM40. In E3 carriers, the presence of the long variant of TOMM 40 increased $\mathrm{AD}$ risk to a level comparable with that for $\mathrm{E} 4$ carriers (see Figure 1, "TOMM40 in Alzheimer's disease").

Overall, carriers of the long form of the variant had nearly $50 \%$ higher risk of developing disease than short-form carriers.

Roses then examined the relationship between the TOMM40 length polymorphism and age of onset in E3 homozygotes, in which the effect of TOMM 40 could be studied independently of E4.

"When the long form of TOMM40 is present in patients with E3, it appears to cause an average age of onset similar to that in $\mathrm{E} 4$ carriers, at about age 70," said Roses. In contrast, E3 patients who had only the short form of TOMM40 had an average age of onset of $78(p=0.03)$. 


\section{Doubting TOMM}

Roses' findings have not yet undergone peer review, and as a result researchers polled by SciBX had mixed opinions on whether the findings would stand up to scrutiny.

Dale Schenk, EVP and CSO of Elan Corp. plc, said Roses' hypothesis could explain variation in AD risk independently of E4, "which is why it's so interesting and provocative." However, Schenk cautioned that the TOMM40 discovery must be independently validated before gaining acceptance in the AD community.

"Almost any genetic finding needs to be replicated before other geneticists buy into it," Schenk told SciBX.

Last month, Johnson \& Johnson said it will form a new company
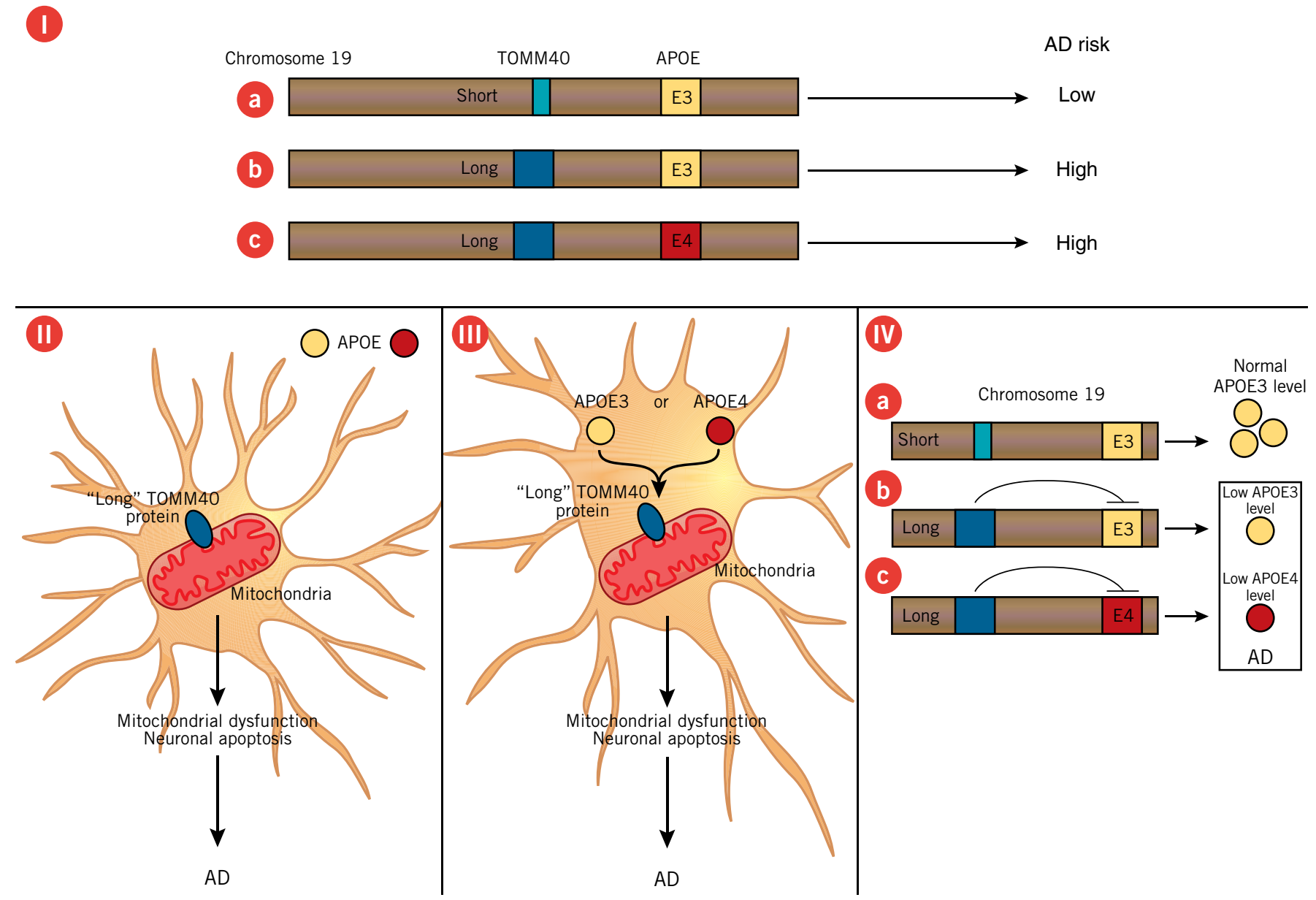

Figure 1. TOMM40 in Alzheimer's disease. At the International Conference on Alzheimer's Disease in Vienna last month, Allen Roses presented genetic evidence that sequence length variation within the TOMM40 (translocase of outer mitochondrial membrane 40 homolog) gene affects AD risk. The TOMM40 gene, which encodes a mitochondrial protein, lies on chromosome 19 adjacent to the gene encoding apolipoprotein E (APOE), a well-known AD risk factor. DNA sequencing of 340 individuals revealed two forms of a noncoding region within the TOMM40 gene.

(I) The most common form contains a short sequence repeat and correlates with an average age of AD onset of 78 years in carriers of the AD-neutral $A P O E$ allele $E 3$ [a], whereas an alternative, longer repeat sequence correlates with average age of $A D$ onset of 70 years in $E 3$ carriers $(n=105, p<0.03)[b]$. The long form is also present in nearly all carriers of APOE allele $E 4$ [c], which was previously shown by Roses to correlate with early $A D$ onset.

Three possible mechanisms could explain the contribution of TOMM40 to AD.

(II) APOE functions outside of the neuron, physically separated from an abnormal form of TOMM40. Abnormal TOMM40 could lead to mitochondrial dysfunction, apoptosis and eventually AD in an APOE-independent manner.

(III) Alternatively, intracellular APOE protein and TOMM40 protein could interact at the mitochondrial surface to disrupt mitochondrial metabolism, leading to apoptosis and AD.

(IV) Finally, length variation in the TOMM40 gene could influence the expression of the adjacent APOE gene. The short form of TOMM4O could lead to strong transcription of $A P O E$ and thus normal E3 protein levels that protect against disease [a]. However, the long form of TOMM40 could dampen expression of $E 3$ [b] or $E 4$ [c], leading to inadequate APOE protein levels and subsequently to AD. 


\section{TARGETS \& MECHANISMS}

to acquire the rights of Elan to an $\mathrm{AD}$ immunotherapy program that is partnered with Wyeth. The program includes four products targeting $\beta$-amyloid (A $\beta)$.

Rudolph Tanzi, professor of neurology at Massachusetts General Hospital, doubts that TOMM40 is a significant player in AD. He noted that many other sequence variants besides the TOMM40 length variant are tightly linked with the APOE locus and that Roses' methods don't conclusively prove that the length variant causes accelerated disease onset.

Instead, Tanzi suggested the apparent association between $\mathrm{AD}$ and TOMM40 variants could be a statistical fluke due to small sample size or the powerful effect of E4 and a counteracting effect by E2, another $A P O E$ variant that protects against $\mathrm{AD}$.

"I honestly think that it's nothing more than a reflection of its chromosomal association with APOE," said Tanzi.

Roses countered that both he and GSK had collectively sequenced a sufficient number of patients to dispel statistical doubts. He said the conclusions hold up even when the effect of E2 is excluded.

Alison Goate, professor of genetics at Washington University in St. Louis, told SciBX she thinks Roses' data corroborate earlier genomewide association studies, but she was cautious about his interpretation.

"People have previously found polymorphisms in TOMM40, but it wasn't possible to disentangle those variants from the correlation with E4," she said.

Goate did note that Roses' deep-sequencing strategy is "the next step forward," giving the clearest picture yet of variation near the APOE locus and thus teasing out genetic effects that are hard to find using conventional genomewide association analysis.

"When you do deep sequencing, you find all

\section{"Nobody can jump to a solid conclusion at this point, but I think that TOMM40 is a very good candidate gene." \\ -Chang-En $Y u$, University of Washington School of Medicine}

\section{$\mathrm{AD}$ (see Figure 1.II)}

Roses favors the idea that TOMM40 interacts with an intracellular, neuronal form of APOE that has been implicated in AD by researchers at the Gladstone Institute of Neurological Disease. According to this theory, defective TOMM40 is harmful in its own right but could also enhance the toxic effects of neuronal APOE, leading to mitochondrial dysfunction, apoptosis and neuron loss (see Figure 1.III).

Although the length polymorphism occurs in a noncoding region of TOMM40, Roses thinks the polymorphism could affect TOMM40 mRNA or protein levels, which in turn could affect mitochondrial activity and TOMM40's interactions with APOE.

"We and a couple of other labs have shown that E3 and E4 bind to the outer mitochondrial membrane by differentially binding to TOMM40," said Roses. Thus, he believes that TOMM40 protein could be a direct player in mitochondrial dysfunction in AD.

An alternative explanation may be that variations in TOMM40 influence the expression of the nearby APOE gene through a knock-on effect see Figure 1.IV).

The possibility that TOMM40 polymorphisms affect APOE gene expression is "a much more reasonable argument," said Schenk. "It's been known for some time that at the transcriptional and translational level, there are differences in APOE expression" caused by nearby DNA.

Indeed, Yu's team recently reported that TOMM40 polymorphisms correlated with different levels of $A P O E$ mRNA and protein in postmortem samples from $\mathrm{AD}$ patients. ${ }^{4}$

Goate said her team has also seen TOMM40 mutations affecting levels of $A \beta$, the aggregation-prone protein fragment most closely associated with $\mathrm{AD} .^{5}$

"I'm agnostic about the mechanism" of TOMM40's role in AD, said Goate. "But I certainly believe that there's something more to it than APOE."

Roses' team at Duke is testing the three possibilities by examining mitochondrial activity and APOE expression in mice bearing TOMM40 transgenes either together or separately from $A P O E$ transgenes.

Chang-En Yu, associate professor of gerontology and geriatric medicine at the University of Washington School of Medicine, leaned more into Roses' camp. "Though people think E4 is the main genetic cause of $\mathrm{AD}$, we cannot completely explain $\mathrm{AD}$ risk" using $\mathrm{E} 4$ alone, he said.

In 2007, Yu and colleagues reported that TOMM40 polymorphisms were tightly linked to higher $\mathrm{AD}$ risk in Caucasians. ${ }^{2}$ Earlier this year, a study by Japanese researchers showed similar results in Japanese AD patients. $^{3}$

"Nobody can jump to a solid conclusion at this point, but I think that TOMM40 is a very good candidate gene," said Yu.

\section{Mechanism questions}

Even if the genetic association between TOMM40 and AD is validated, how the gene influences AD remains opaque. Several models are possible, each with different implications about targeting TOMM40 with therapeutics.

Because TOMM40 helps transport other proteins into the mitochondrial interior, defects in TOMM40 could compromise mitochondrial function, weakening neuronal health independently of APOE's role in

\section{The mitochondrial link}

If TOMM40 protein is indeed a direct player in $\mathrm{AD}$, mitochondria may be key therapeutic targets.

"Roses' data imply that a mitochondrial protein may have relevance to AD," said Medivation Inc. CMO Lynn Seely. The company's Dimebon latrepirdine is an oral small molecule believed to enhance mitochondrial function. The compound is in a Phase III AD trial and is partnered with Pfizer Inc.

Roses believes that the best way to counteract TOMM40 and $A P O E$ dysfunction is to increase mitochondrial numbers. Peroxisome proliferation-activated receptor- $\gamma$ (PPARG; PPAR $\gamma$ ) agonists could serve this purpose because they also stimulate mitochondrial proliferation.

An earlier effort to test whether PPAR $\gamma$ agonists could do this was run by GSK. There, Roses oversaw Phase II and III trials of the PPAR $\gamma$ agonist Avandia rosiglitazone to treat moderate-to-advanced AD. Earlier this year, GSK reported that an extended-release formulation of 


\section{TARGETS \& MECHANISMS}

Avandia failed a Phase III trial, and the company halted further development of the drug in AD.

Roses attributed the clinical setback to a patient population that was too far advanced in the disease process to benefit from higher numbers of mitochondria. "As a treatment where the cells are already dead or dying, it won't be as effective," he noted.

Zinfandel, where Roses is president and CEO, is collaborating with clinics in Russia to identify a large number of middle-aged TOMM40 long-form carriers and noncarriers who have not yet developed $\mathrm{AD}$. Roses plans to treat these individuals with low doses of a to-be-determined PPAR $\gamma$ agonist over several years. If his theory is correct, TOMM40 longform carriers will develop $\mathrm{AD}$ more quickly than noncarriers, but disease progression in both groups could be slowed by the PPAR $\gamma$ agonist.

The study should thus test two hypotheses: the role of long-form TOMM40 in accelerated AD progression and the ability of PPAR $\gamma$ agonists to ameliorate $\mathrm{AD}$.

Roses said that running such a trial in Russia is attractive because of the country's relative genetic homogeneity and good medical records. He also noted the regulatory challenge of doing a prophylactic trial using an unvalidated biomarker and a therapeutic agent with a history of adverse cardiovascular events at higher doses.

"Getting the FDA to do something that hasn't been done before is very difficult," he said.

Roses has filed patents for the use of TOMM40 repeat polymorphisms to predict AD risk and on new methods of identifying genetic diseases used in his studies. The diagnostic technology is being licensed to Shiraz Pharmaceuticals Inc., another company headed by Roses.

Osherovich, L. SciBX 2(30); doi:10.1038/scibx.2009.1165 Published online Aug. 6, 2009

\section{REFERENCES}

1. Osherovich, L. SciBX 2(24); doi:10.1038/scibx.2009.962

2. Yu, C.-E. et al. Genomics 89, 655-665 (2007)

3. Takei, N. et al. Genomics 93, 441-448 (2009)

4. Bekris, L. et al. Am. J. Med. Genet. B Neuropsychiatr. Genet.; published online June 24, 2009; doi:10.1002/ajmg.b.30993

5. Kauwe, J.S.K. et al. Neurogenetics 10, 13-17 (2009)

\section{COMPANIES AND INSTITUTIONS MENTIONED}

Deane Drug Discovery Institute at Duke University, Durham, N.C. Elan Corp. plc (NYSE:ELN), Dublin, Ireland

Eli Lilly and Co. (NYSE:LLY), Indianapolis, Ind.

Gladstone Institute of Neurological Disease, San Francisco, Calif. GlaxoSmithKline plc (LSE:GSK; NYSE:GSK), London, U.K. Johnson \& Johnson (NYSE:JNJ), New Brunswick, N.J.

Lilly Research Laboratories, Indianapolis, Ind.

Massachusetts General Hospital, Boston, Mass.

Medivation Inc. (NASDAQ:MDVN), San Francisco, Calif.

Pfizer Inc. (NYSE:PFE), New York, N.Y.

Shiraz Pharmaceuticals Inc., Durham, N.C.

University of Washington School of Medicine, Seattle, Wash. Washington University in St. Louis, St. Louis, Mo.

Wyeth (NYSE:WYE), Madison, N.J.

Zinfandel Pharmaceuticals Inc., Durham, N.C. 